\title{
Construindo um instrumento de avaliação da percepção ambiental associada aos impactos ambientais das ações cotidianas
}

Buildling an instrument for assessing the environmental perception associated with the environmental impacts of daily actions

Construcción de un instrumento para evaluar la percepción ambiental asociada a los impactos ambientales de las acciones cotidianas

Recebido: 27/04/2021 | Revisado: 05/05/2021 | Aceito: 07/05/2021 | Publicado: 21/05/2021

\author{
Lilian Janine de Barros Beckmann \\ ORCID: https://orcid.org/0000-0002-3738-9967 \\ Universidade Federal do Pampa, Brasil \\ E-mail: lilianbeckmann@unipampa.edu.br \\ Carlos Maximiliano Dutra \\ ORCID: https://orcid.org/0000-0003-4743-874X \\ Universidade Federal do Pampa, Brasil \\ E-mail: carlosdutra@unipampa.edu.br
}

\begin{abstract}
Resumo
Nos últimos anos a degradação ambiental se intensificou e a finitude dos recursos naturais tornou-se mais evidente. $\mathrm{O}$ desenvolvimento econômico e tecnológico nem sempre está em harmonia com o meio ambiente, gerando uma série de danos ambientais que precisam ser compreendidos e admitidos. Neste trabalho aborda-se a construção de um instrumento para avaliar a percepção do impacto ambiental das atividades cotidianas dos indivíduos. Foi elaborado um questionário que avalia a percepção de impacto ambiental relacionada aos aspectos de alimentação, habitação, transporte, consumo de água, consumo energia elétrica, e aquisição de vestuário. Após foi realizado o processo de validação do questionário que resultou em uma questão descritiva e quatorze questões objetivas sobre como as diversas atividades cotidianas podem impactar o meio ambiente numa escala de Likert de 1 a 10 . O questionário foi aplicado a estudantes de um curso de graduação e os resultados apontaram coerência interna registrando como principais impactos reconhecidos: a produção de lixo, os desperdícios de água e luz, e a poluição do ar. O instrumento de avaliação de impacto ambiental das ações cotidianas constitui-se em uma ferramenta que pode ser utilizada no contexto escolar na abordagem de temas relacionados a questão ambiental para promover uma discussão sobre os impactos ambientais produzidos por nossas ações individuais; e também pode ser utilizado para a avaliação de atividades pedagógicas relacionadas com a Educação Ambiental através da aplicação antes e após a aplicação dessas atividades.
\end{abstract}

Palavras-chave: Percepção ambiental; Impacto ambiental; Avaliação ambiental.

\begin{abstract}
In recent years, environmental degradation has intensified and the finitude of natural resources has become more evident. Economic and technological development is not always in harmony with the environment, generating a series of environmental damages that need to be understood and admitted. This work addresses the construction of an instrument to assess the perception of the environmental impact of individuals' daily activities. A questionnaire was prepared to assess the perception of environmental impact related to the aspects of food, housing, transportation, water consumption, electricity consumption, and clothing purchase. Afterwards, the questionnaire validation process was carried out, which resulted in a descriptive question and fourteen objective questions about how the various daily activities can impact the environment on a Likert scale from 1 to 10. The questionnaire was applied to students of an undergraduate course and the results pointed out internal coherence, registering as main recognized impacts: the production of garbage, waste of water and light, and air pollution. The instrument for assessing the environmental impact of everyday actions is a tool that can be used in the school context to address issues related to the environmental issue to promote a discussion on the environmental impacts produced by our individual actions; and it can also be used for the evaluation of pedagogical activities related to Environmental Education through the application before and after the application of these activities.
\end{abstract}

Keywords: Environmental perception; Environmental impact; Environmental assessment.

\section{Resumen}

En los últimos años, la degradación ambiental se ha intensificado y la finitud de los recursos naturales se ha vuelto más evidente. El desarrollo económico y tecnológico no siempre está en armonía con el medio ambiente, generando una 
serie de daños ambientales que deben ser comprendidos y admitidos. Este trabajo aborda la construcción de un instrumento para evaluar la percepción del impacto ambiental de las actividades diarias de las personas. Se elaboró un cuestionario para evaluar la percepción de impacto ambiental relacionado con los aspectos de alimentación, vivienda, transporte, consumo de agua, consumo eléctrico y compra de ropa. Posteriormente, se llevó a cabo el proceso de validación del cuestionario, que resultó en una pregunta descriptiva y catorce preguntas objetivas sobre cómo las diferentes actividades diarias pueden impactar el medio ambiente en una escala Likert del 1 al 10. El cuestionario se aplicó a estudiantes de un curso de pregrado. los resultados señalaron coherencia interna, registrando como principales impactos reconocidos: la producción de basura, el desperdicio de agua y luz y la contaminación del aire. El instrumento de evaluación del impacto ambiental de las acciones cotidianas es una herramienta que se puede utilizar en el contexto escolar para abordar temas relacionados con el tema ambiental para promover una discusión sobre los impactos ambientales producidos por nuestras acciones individuales; y también se puede utilizar para la evaluación de actividades pedagógicas relacionadas con la Educación Ambiental a través de la aplicación antes y después de la aplicación de estas actividades.

Palabras clave: Percepción ambiental; Impacto ambiental; Evaluación ambiental.

\section{Introdução}

Nos últimos anos a degradação ambiental se intensificou e a finitude dos recursos naturais tornou-se mais evidente (Marques \& Xavier, 2019). O desequilíbrio ambiental é um dos temas mais discutidos na contemporaneidade, e os impactos ambientais evidenciam a dissimetria entre o comportamento humano e o meio ambiente (Zacarias \& Higuchi, 2017; Oliveira e Brasil, 2020). Mudanças no entendimento destas questões ambientais de cada indivíduo são fundamentais para a diminuição da destruição do planeta (Amaral, Arantes \& Bernardes, 2020).

A educação ambiental deve almejar o estímulo a mudanças de hábitos, comportamentos sustentáveis e, principalmente, conscientizar a população (Royer \& Petrik, 2019; Santos, Costa \& Melo Souza, 2020). A prática para a sustentabilidade passa a ser introduzida no processo educacional, incluindo a educação superior, para que possamos formar comunidades mais cooperativas e com desenvolvimento sustentável, através de uma convivência harmoniosa entre homem e meio ambiente no Planeta (Mota et al, 2020; Cerqueira et al, 2020); superando assim, o analfabetismo ambiental (Junior, 2018). Estratégias educativas devem ser estimuladas na abordagem da temática ambiental, a fim de refletir sobre a importância na redução dos impactos ambientais (Nunes, França \& Paiva, 2017). Além de metodologias de ensino envolvendo a educação ambiental é interessante avaliar a percepção ambiental dos alunos como uma forma de acessar os conhecimentos prévios e consciência ambiental. Neste trabalho, propomos um instrumento de pesquisa na forma de questionário para avaliação da percepção ambiental frente ao impacto causado pelas atitudes cotidianas e várias formas de consumo.

\section{Metodologia}

O desenvolvimento de um questionário como instrumento de pesquisa envolve a elaboração das questões e a realização da validação do instrumento tornando o apto à aplicação (Cohen et al., 2007; Pasquali, 2010).

A pegada ecológica (PE) mostra-se como eficiente estratégia na mudança da percepção do indivíduo quanto as questões ambientais (Lamin Guedes, 2015; Marques \& Xavier, 2020). A pegada ecológica, como recurso didático tem sido utilizada em diferentes ambientes, como no ensino fundamental (Berté, 2019; Batista et al, 2019), em cursos de pós-graduação (Paulista et al., 2018), no ensino médio (Meyer, 2018) e em Universidades (Lied et al, 2019; Collins et al 2018). O uso da ferramenta PE permite calcular os recursos naturais que uma pessoa, cidade, país ou organização utilizam para manter seu estilo de vida no período de um ano, proporcionando um método prático de medir as marcas que deixamos no Planeta, trazendo nova forma de avaliar a sustentabilidade (Rees \& Wackernagel, 1996; Lamim-Guedes, 2015). O questionário piloto foi elaborado correlacionando o consumo com cinco temas que compõe os núcleos básicos da ferramenta Pegada Ecológica (PE): alimentação, habitação, transporte, bens e serviços (Fonseca \& Oliveira, 2013; Berte, 2019; Leite at al 2020). O uso da energia elétrica e a aquisição de vestuário foram utilizados como representantes de serviços e bens, respectivamente (Almeida, Scatena \& Luz 2017; 
Turmina et al, 2018; Moro \& Paulino, 2020). As questões específicas do questionário piloto foram formuladas relacionando o consumo com maior ou menor intensidade dos impactos ambientais dentro de cada tema proposto. Assim, 20 questões foram elaboradas (quatro para cada tema), sendo que para cada questão era possível uma resposta em escala linear de um a 10 (a) e uma na forma discursiva (b). Na escala linear, foi considerado 1 como baixo nível de impacto ambiental e 10 como alto nível de impacto ambiental. Ainda, buscou-se a forma adequada para que os respondentes não fossem inclinados a procurar questões certas ou erradas. Após a construção do questionário piloto foi desenvolvida a etapa de validação.

Para que um questionário desenvolvido seja considerado eficaz para a realização de pesquisas científicas, a validação é um precedente importante; fundamentando-se numa sequência de procedimentos metodológicos que permitem comprovar a confiabilidade do instrumento (Cohen et al., 2007; Pasquali, 2009; Pimentel, 2018). Para tal, diferentes tipos de validação de questionário são encontrados na literatura, tais como: validade de construto, validade de critério, validação de face, validação semântica, validação ecológica, validade preditiva, validade interna, validade externa (Echevarria-Guanilo, Gonçalves \& Romanoski, 2019; Pasquali, 2013). Na perspectiva de DeVellis (2003) há três tipos principais de validação, os quais são validação de conteúdo, validação de critério e validação de construto. Nas pesquisas da área de educação, quando se aborda validade de instrumento, a validação de conteúdo aparece de forma mais significativa (Siqueira e Marques, 2018; Vizzotto \& Mackedanz, 2019; Schwantz et al, 2019; Vilarinho e Monteiro, 2019; Silva et al 2020). Para o estabelecimento da validade de conteúdo, podem ser empregadas como estratégias a revisão de literatura e o julgamento de profissionais com experiência na área (Alexandre \& Coluci, 2011; Roisemam, 2008; Braga et al, 2018; Silva et al, 2020). Como não existe um teste estatístico específico para avaliação da validade de conteúdo, geralmente utiliza-se uma abordagem qualitativa, por meio da avaliação de um grupo de especialistas (Souza et al., 2017; Oliveira \& Aguiar et al, 2018; Silva et al, 2020). A validação de semântica, de conteúdo e a validade de face tem se mostrado efetivas nas pesquisas com temática ambiental (Jesus e Bedritichuk 2018; Carvalho \& Andreto, 2020). A validade de face permite garantir que o instrumento seja compreendido pelos participantes, não gerando dúvidas capazes de impedir a obtenção dos objetivos (Natalli et al, 2020; Luis et al, 2012). A validação de face indica se o instrumento responde aos objetivos ao qual se propôs (Pimentel, 2018). A validação semântica refere-se a linguagem utilizada e confere a representatividade desta linguagem em relação ao público abordado (Guedes, 2016; Magalhães Júnior et al, 2020). A validade semântica tem como objetivo diminuir as dubiedades do público testado e determinar a clareza na linguagem das questões, envolve a compreensão dos itens pela população a qual irá responder o questionário (Santos, 2016; Freitas et al, 2020).

Para o processo de validação do questionário optou-se por focar na validação de conteúdo e validação semântica segundo as definições de Cohen et al. (2007), Coluci, Alexandre e Milani (2015), Leite et al (2018) e Pimentel (2018).

A validação de conteúdo e semântica do questionário foi realizada de maneira independente por especialistas, a partir da avaliação crítica deste e dos critérios que orientaram a sua construção (Magalhaes Jr et al 2020, Natalli et al, 2020). O atributo clareza foi utilizado para a validação de semântica e os atributos objetividade, relevância e simplicidade foram utilizados para a validação de conteúdo (Pasqualli, 2009). Para tal, foi elaborado e utilizado um formulário de validação com a possibilidade de concordância com os atributos para cada uma das questões (Pimentel 2018, Natalli et al, 2020; Magalhães Jr. et al, 2020). A validação do instrumento foi realizada por três professores especialistas na área de ensino e educação ambiental, com mais de 15 anos de experiência na docência. O critério utilizado para a seleção dos especialistas foi o conhecimento na temática da pesquisa e o tempo de atuação na área (Luis et al, 2012; Torres \& Fermam, 2016; Leite et al, 2018). O formulário de validação, ficha de instruções e o link do questionário foram enviados para as especialistas através de e-mail.

Após a devolução do questionário piloto devidamente analisado e preenchido pelos especialistas, as informações foram verificadas e compiladas em planilha única. As questões foram reestruturadas a partir do percentual de concordância das 
respostas dos avaliadores (Coluci, Alexandre \& Milani, 2015; Cunha, Neto \& Stackfleth, 2016; Nunes et al, 2019 e Silva et al, 2020). O percentual de concordância é representado pela seguinte formula:

$$
\% \text { de concordância }=\frac{\text { número de participantes que concordam com o item }}{\text { número total de participante }} \times 100
$$

O nível de concordância dos avaliadores quanto aos atributos em cada questão está apresentado na tabela 1 . A discordância dos avaliadores foi maior na validação de semântica (atributo clareza), mas com sugestões de pequenos ajustes, substituindo expressões para facilitar o entendimento dos respondentes. Quanto ao conteúdo, houve apenas a divergência de um avaliador em uma questão (Tabela 1).

Tabela 1: Percentual de concordância dos especialistas.

\begin{tabular}{|c|c|c|c|c|}
\hline \multirow{2}{*}{ Questões } & \multirow{2}{*}{$\begin{array}{c}\begin{array}{c}\text { Validação } \\
\text { de }\end{array} \\
\text { semântica }\end{array}$} & \multicolumn{3}{|c|}{ Validação de conteúdo } \\
\hline & & Objetividade & Relevância & Simplicidade \\
\hline 1 & $66 \%$ & $100 \%$ & $100 \%$ & $100 \%$ \\
\hline 2 & $66 \%$ & $100 \%$ & $100 \%$ & $100 \%$ \\
\hline 3 & $66 \%$ & $100 \%$ & $100 \%$ & $100 \%$ \\
\hline 4 & $66 \%$ & $100 \%$ & $100 \%$ & $100 \%$ \\
\hline 5 & $66 \%$ & $100 \%$ & $100 \%$ & $100 \%$ \\
\hline 6 & $100 \%$ & $100 \%$ & $100 \%$ & $100 \%$ \\
\hline 7 & $100 \%$ & $100 \%$ & $100 \%$ & $100 \%$ \\
\hline 8 & $66 \%$ & $100 \%$ & $100 \%$ & $100 \%$ \\
\hline 9 & $100 \%$ & $100 \%$ & $100 \%$ & $100 \%$ \\
\hline 10 & $100 \%$ & $100 \%$ & $100 \%$ & $100 \%$ \\
\hline 11 & $100 \%$ & $100 \%$ & $100 \%$ & $100 \%$ \\
\hline 12 & $100 \%$ & $100 \%$ & $100 \%$ & $100 \%$ \\
\hline 13 & $100 \%$ & $100 \%$ & $100 \%$ & $100 \%$ \\
\hline 14 & $100 \%$ & $100 \%$ & $100 \%$ & $100 \%$ \\
\hline 15 & $100 \%$ & $100 \%$ & $100 \%$ & $100 \%$ \\
\hline 16 & $100 \%$ & $100 \%$ & $100 \%$ & $100 \%$ \\
\hline 17 & $100 \%$ & $100 \%$ & $66 \%$ & $100 \%$ \\
\hline 18 & $100 \%$ & $100 \%$ & $100 \%$ & $100 \%$ \\
\hline 19 & $100 \%$ & $100 \%$ & $100 \%$ & $100 \%$ \\
\hline 20 & $100 \%$ & $100 \%$ & $100 \%$ & $100 \%$ \\
\hline
\end{tabular}

Fonte: Autores (2021).

As alterações sugeridas foram realizadas, com o intuito de que o instrumento ficasse adequado para o que pretende medir. A validação de semântica e de conteúdo foi importante para refinar as questões e determinar a inclusão delas no questionário pré-teste (Pedreira et al, 2016). As questões discursivas, elaboradas no questionário inicial, foram reduzidas a uma questão para diminuir o tempo de preenchimento. De acordo com Nobre (2012) e Pasqualli (2013) questões curtas e sem um número muito extenso de itens, podem evitar problemas como fadiga/cansaço/apatia em responder até o final.

O questionário resultante do processo de validação é apresentado no Quadro 1 e constitui-se num instrumento com 14 questões objetivas e uma questão descritiva, além de duas perguntas sobre o perfil dos respondentes. As questões objetivas foram limitadas a 14, três para cada um dos temas (alimentação, habitação, transporte e serviço) e duas para o tema Bens. Para cada uma destas questões, foi mantida a possibilidade de resposta em escala linear de um a 10, sendo considerado 1 como baixo nível 
de impacto ambiental e 10 como alto nível de impacto ambiental. A questão aberta (discursiva) teve intuito de dimensionar qual a compreensão e vivência dos estudantes sobre o tema.

Quadro 1 - Questionário “consumo x impactos ambientais” após restruturação a partir da validação de conteúdo e semântica e pré-teste.

\begin{tabular}{|c|c|}
\hline QUESTÕES - CONSUMO X IMPACTOS AMBIENTAIS & RESPOSTAS \\
\hline Idade & anos \\
\hline Gênero & Feminino, masculino, outro \\
\hline $\begin{array}{l}\text { 1. Cite quais as atividades e práticas de consumo de bens e de serviços do dia a dia que } \\
\text { impactam NEGATIVAMENTE no Meio Ambiente }\end{array}$ & Resposta aberta \\
\hline 02. O desperdício de alimentos. (tema alimentação) & Resposta em escala Likert de 1 a 10 \\
\hline 03. Uma dieta rica em consumo de carne bovina (mais de $3 x$ por semana). (tema alimentação) & Resposta em escala Likert de 1 a 10 \\
\hline $\begin{array}{l}\text { 04. Consumir mais produtos industrializados do que os produtos naturais produzidos na região. } \\
\text { (tema alimentação) }\end{array}$ & Resposta em escala Likert de 1 a 10 \\
\hline 05. Manter a torneira aberta enquanto escova os dentes. (tema habitação) & Resposta em escala Likert de 1 a 10 \\
\hline 06.Tomar banho de forma demorada (mais de 15 min.). (tema habitação) & Resposta em escala Likert de 1 a 10 \\
\hline $\begin{array}{l}\text { 07. Não observar o consumo de energia dos produtos/equipamentos no momento da compra. } \\
\text { (tema serviço) }\end{array}$ & Resposta em escala Likert de 1 a 10 \\
\hline 08. Usar o ar-condicionado e aquecedores. (tema serviço) & Resposta em escala Likert de 1 a 10 \\
\hline $\begin{array}{l}\text { 09. Gestos como manter as luzes acessas em ambientes que não estão sendo ocupados e não } \\
\text { desligar da tomada aparelhos com recurso standby, depois de utilizá-los. (tema serviço) }\end{array}$ & Resposta em escala Likert de 1 a 10 \\
\hline 10. Adquirir produtos de vestuário (roupas e calçados) muito além da necessidade. (tema bens) & Resposta em escala Likert de 1 a 10 \\
\hline 11. Utilizar sacolas plásticas descartáveis nas compras. (tema bens) & Resposta em escala Likert de 1 a 10 \\
\hline 12. Não guardar o lixo para descartá-lo em local adequado. (tema habitação) & Resposta em escala Likert de 1 a 10 \\
\hline 13. Não usar a bicicleta ou o transporte público, quando for possível. (tema transporte) & Resposta em escala Likert de 1 a 10 \\
\hline 14. Não fazer uso de carona compartilhada. (tema transporte) & Resposta em escala Likert de 1 a 10 \\
\hline 15. Viajar de avião. (tema transporte) & Resposta em escala Likert de 1 a 10 \\
\hline
\end{tabular}

Fonte: Autores (2021).

\section{Resultados e Discussão}

O questionário validado foi aplicado junto a uma turma de graduação do curso de pedagogia de uma instituição privada da cidade de Santa Maria-RS. Os respondentes não tiveram dificuldades em relação a semântica e os dados mostraram-se válidos. O tempo médio para responder o questionário foi de 15 minutos. Sabe-se que questionários longos acabam apresentando decréscimo na qualidade das respostas (Nobre, 2012).

A partir da primeira questão de caráter descritivo foi possível verificar as atividades/hábitos de consumo que os estudantes consideram que mais impactam negativamente causando a degradação do ambiente, apresentadas na Figura 1 através de nuvem de palavras. 
Figura 1: Nuvem de palavras com as expressões dos discentes para as ações que mais causam degradação no meio ambiente.

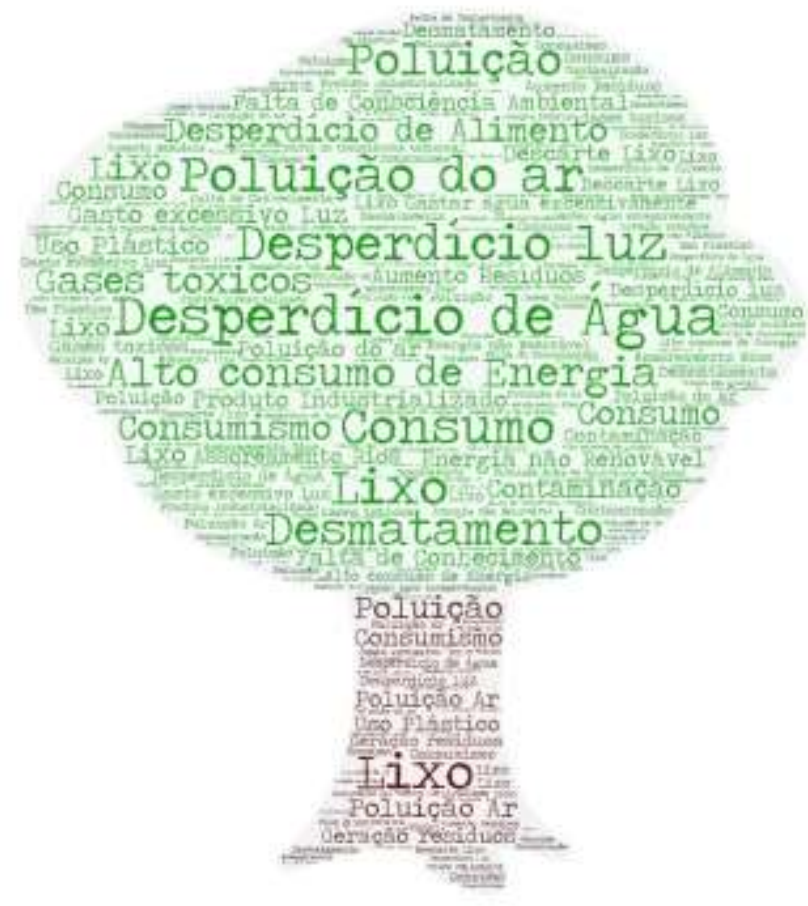

Fonte: Autores (2021).

Em ordem decrescente as expressões mais citadas foram: Desperdício de água, desperdício de luz, alto consumo de energia, poluição do ar, consumo, produção de lixo, desmatamento, desperdício de alimento e poluição. Estes dados manifestam que a compreensão do tema ambiental ainda se limita a questões há muito debatidas, sem repensar o processo de transformação das sociedades. Sendo necessário um repensar sobre a complexidade ambiental (Ribeiro, Coutinho e Boer, 2020).

Com base nas respostas das questões objetivas de 2 a 15 foi possível calcular o valor médio e desvio padrão para mensurar o impacto ambiental percebido em cada situação, conforme Tabela 2.

Tabela 2: Resultados das questões objetivas.

\begin{tabular}{|l|l|l|l|l|l|l|l|}
\hline Questão & Média & $\begin{array}{l}\text { Desvio- } \\
\text { padrão }\end{array}$ & ordem & Questão & Média & $\begin{array}{l}\text { Desvio } \\
\text { padrão }\end{array}$ & ordem \\
\hline 2 & 6,9 & 2,8 & 10 & 9 & 7,1 & 1,8 & 9 \\
\hline 3 & 6,1 & 2,7 & 12 & 10 & 6,1 & 2,5 & 13 \\
\hline 4 & 7,3 & 2,1 & 7 & 11 & 9,4 & 1,2 & 1 \\
\hline 5 & 8,5 & 2,0 & 4 & 12 & 8,8 & 1,7 & 3 \\
\hline 6 & 8,9 & 1,5 & 2 & 13 & 7,7 & 2,1 & 5 \\
\hline 7 & 7,1 & 1,8 & 8 & 14 & 7,3 & 2,6 & 6 \\
\hline 8 & 6,8 & 2,8 & 11 & 15 & 5,3 & 2,4 & 14 \\
\hline
\end{tabular}

Fonte: Autores (2021).

Através dessa compilação dos dados pode-se estabelecer uma ordem dos fatores que tem maior impacto ambiental do ponto de vista da percepção dos participantes do questionário, sendo esta a ordem decrescente conforme a média dos indicadores 
de avaliação da percepção de impacto ambiental para os cinco fatores considerados mais impactantes: (1) sacolas plásticas; (2) banho demorado; (3) descarte não adequado do lixo; (4) torneira aberta ao escovar dentes; e (5) não usar bicicleta ou transporte público. Correlacionando essas percepções de impacto ambiental vinda das questões objetivas com as vindas da questão descritiva, verifica-se que os impactos apontados em $1^{\mathrm{a}}$ e $3^{\mathrm{a}}$ colocações estão relacionados com a "produção de lixo" destacada na questão descritiva; percebe-se que o impacto apontado em $2^{\text {a }}$ colocação está relacionado com o "desperdício de luz" e "alto consumo de energia" destacados na questão descritiva, e além disso esse impacto conjuntamente com o impacto em $4^{\mathrm{a}}$ colocação estão relacionados com o "desperdício de água" também destacado na questão descritiva. Por fim o impacto na $5^{\text {a }}$ colocação está relacionado com a "poluição do ar" igualmente destacada. Considerando a Tabela 2 observa-se que nas três últimas colocações tem-se as questões (3) alimentação carne bovina (10) adquirir vestuário e (15) viajar de avião essas situações não foram apontadas ou tiveram pouca ocorrência na parte descritiva do questionário. A avaliação da percepção de impacto ambiental realizada através da parte objetiva do questionário demonstrou-se compatível com a obtida com a questão descritiva demonstrando a coerência dos resultados da aplicação.

\section{Considerações Finais}

A degradação do meio ambiente pelas atividades humanas sobretudo as de exploração do meio natural para obtenção de energia ou para uso industriais são constantemente retratadas como as principais causas de impacto ambiental necessário para a vida moderna com desenvolvimento econômico e tecnológico. Pelo fato desses impactos beneficiarem nossas vidas e realizados por empresas e governos acabamos por não nos incluirmos ou incluirmos nossas ações cotidianas como fonte de impacto ambiental. Daí a necessidade do desenvolvimento de uma consciência ambiental através da inserção da Educação Ambiental como tema transversal na Educação Básica e também em alguns cursos de graduação principalmente os relacionados a questão ambiental e/ou os cursos de licenciatura para formação de novos profissionais comprometidos com o desenvolvimento sustentável. Em especial, os professores têm um papel importante neste processo de formação da consciência ambiental que se consolidará através de uma cultura comprometida com o meio ambiente.

No processo de desenvolvimento da Educação Ambiental tão importante como a realização de atividades pedagógicas de conscientização ambiental, se faz necessário a construção de instrumentos que avaliem a percepção de impacto ambiental do estudante. Ao longo do presente trabalho foi elaborado um instrumento de avaliação da percepção ambiental do impacto ambiental das ações diárias dos indivíduos abordando aspectos relacionados com a hábitos de consumo na alimentação, na água, na energia elétrica e outros serviços e bens. O questionário permite elencar as atividades de consumo que mais impactam ambientalmente na percepção dos entrevistados. Na sala de aula, esse instrumento poderá se mostrar útil para introduzir temas relacionados a questão ambiental para que os professores possam desenvolver com maior propriedade a temática do Meio Ambiente, levando em conta os conhecimentos prévios e o nível de consciência dos alunos em relação ao tema expressos nas atitudes diárias.

Para futuras pesquisas pretende-se usar o presente instrumento de avaliação da percepção de impacto ambiental para investigar a mudança de percepção ambiental em decorrência da aplicação de atividades pedagógicas relacionadas com a temática de Educação Ambiental como instrumento de pré e pós-teste; procurando avaliar o potencial das práticas ambientais em transformar a consciência ambiental dos educandos.

\section{Referências}

Alexandre, N. M. C. \& Coluci, M. Z. O. (2011). Validade de conteúdo nos processos de construção e adaptação de instrumentos de medidas. Revista Ciência \& Saúde Coletiva. 16(7). https://www.scielosp.org/article/csc/2011.v16n7/3061-3068/.

Almeida, R.; Scatena, L. M. \& Luz, M. S. (2017). Percepção Ambiental e Políticas Públicas - Dicotomia e desafios no desenvolvimento da cultura de sustentabilidade. Ambiente \& sociedade. XX(1), 43-64. https://www.redalyc.org/articulo.oa?id=31750848004. 
Amaral, L. L. R., Arantes, G. G. \& Bernardes, M. B. J. (2020). Consumo consciente por meio da educação ambiental na escola. Revista Ensino de Geografia (Recife), 3(1). https://periodicos.ufpe.br/revistas/ensinodegeografia/article/viewFile/244511/34844.

Batista, N. L., Feltrin, T., Cassol, M., Ziembowicz, F. I.,Vargas, L. F., \& Pigatto, M. M. (2019). Uma proposta multidisciplinar para a educação ambiental com alunos de $6^{\circ}$ ano do ensino fundamental em Santa Maria/RS. Vivências: Revista Eletrônica de Extensão da URI. 15(28), 193-205. 10.31512/vivencias.v15i28.28.

Berte, E. A. (2019). Indicadores socioambientais: a pegada ecológica como ferramenta para educação ambiental na escola. Universidade Tecnológica Federal do Paraná. http://repositorio.roca.utfpr.edu.br/jspui/handle/1/12978.

Braga, W. R. O., Moraes, N. R., Baptista, R. D., Putti, F. F., \& Braga Jr, S. S. (2018). A construção da percepção ambiental de estudantes universitários brasileiros. Revista Observatório, 4(3), 1076-1106.https://sistemas.uft.edu.br/periodicos/index.php/observatorio/article/view/5247.

Carvalho, I. K. S. \& Andreto, L. M. (2020). Elaboração e validação de diretrizes de educação em saúde para o ensino médio. Revista Eletrônica Acervo Saúde, 12(6), e3175. https://doi.org/10.25248/reas.e3175.2020.

Cerqueira, F. G., Souza, A. C. S. S., Thompson, B. B., Michalichen, G., Brito, G. G. S., Pereira, M. O. F., Kreczkiuski, C., Canonico, C. M., Porrua, D. A., Biolchi, G., Dias, H. M., Arancibia, I. A. L., Ribeiro, J. C. B., Minozzo, M., Reis, M. E. M., Felipe, N. A., Krefta, S. C., Potrich, M. \& Tessaro, D. (2020). Educação Ambiental - Percepções e reflexões pautadas no projeto "Plante Essa Ideia". Research, Society and Development, 9(9), e746997710. DOI: 10.33448/rsd-v9i9.7710. https://rsdjournal.org/index.php/rsd/article/view/7710.

Cohen, L., Manion, L., \& Morrison, K. (2007). Research methods in education (6a ed.). Routledge.

Collins, A., Galli, A., Patrizi, N. \& Pulselli, F. M. (2018). Learning and teaching sustainability: the contribution of ecological footprint calculators. Journal of Cleaner Production, 174, 1000-1010. https://doi.org/10.1016/j.jclepro.2017.11.024.

Coluci, M. Z. O., Alexandre, N. M. C. \& Milani, D. (2015). Construção de instrumentos de medida na área da saúde. Revista Ciência \& Saúde Coletiva, 20(3):925-936. 10.1590/1413-81232015203.04332013.

Cunha, C. M., Omar P. A. N. \& Stackfleth, R. (2016). Principais métodos de avaliação psicométrica da validade de instrumentos de medida. Revista Atenção Saúde, 14(47),75-83. 10.13037/rbcs.vol14n47.3391.

Devellis, Robert F. (2003). Scale Development - Theory and aplications. (2a ed.). Editora Sage Publications.

Echevarria-Guanilo, M. E., Goncalves, N. \& Romanoski, P. J. (2019). Propriedades psicométricas de instrumentos de medidas: bases conceituais e métodos de avaliação - parte II. Texto \& contexto - enfermagem, 28. http://dx.doi.org/10.1590/1980-265x-tce-2017-0311.

Fonseca, I. \& Oliveira, S. S. (2013). A pegada ecológica como instrumento metodológico na relação meio ambiente e ensino de ciências. Cadernos PDE. I. http://www.diaadiaeducacao.pr.gov.br/portals/cadernospde/pdebusca/producoes_pde/213/2013_uel_cien_artigo_ironice_da_fonseca_mazeto.pdf.

Freitas R., Waechter H., Coutinho S. \& Gubert F. (2020). Validação de aspectos semânticos em diretrizes para elaboração de Materiais Educativos Impressos para Promoção da Saúde: contribuição do Design da Informação. Revista Brasileira de Design da Informação/Brazilian Journal of Information Design. 17(1), 152 - 169. https://infodesign.emnuvens.com.br/infodesign/article/view/759.

Guedes, R. de M. (2016). O princípio da garantia semântica e os estudos da linguagem. 153p. Tese (Doutorado em Ciência da Informação) - Escola de Ciência da Informação, Universidade Federal de Minas Gerais, Belo Horizonte.

Jesus, G. R., \& Bedritichuk, A. G. A. (2018) Autoavaliação institucional: construção e validação de um questionário para o professor. Avaliação. 23(3), 628647. https://doi.org/10.1590/s1414-40772018000300004.

Junior, A. S. V. (2018). Sustentabilidade socioambiental em sala de aula. https://eventos.set.edu.br/index.php/enfope/article/view/8721.

Lamim-Guedes, V. (2015). Pegada ecológica como recurso didático em atividades de educação ambiental on-line. Revista Educação Unisinos, 19(2), 283 a 289. http://revistas.unisinos.br/index.php/educacao/article/view/edu.2015.192.12.

Leite, R., Almeida, G. F., Sousa, A. N., Araújo, A. O., Pereira, D. S. O., Cardoso, E. F., Mendes, H. S. \& Cordato, L. S. S. (2020). The ecological footprint as a comparative instrument of the levels of environmental sustainability in public and private schools in the Municipality of Pombal, Paraíba, Brazil. Research, Society and Development, 9(9). 10.33448/rsd-v9i9.7268. https://rsdjournal.org/index.php/rsd/article/view/7268.

Leite, S. de S., Afio, A. C. E., Carvalho, L. V., Silva, J. M., Almeida, P. C. \& Pagliuca, L. M. F. (2018). Construction and validation of an Educational Content Validation Instrument in Health. Revista Brasileira de Enfermagem. 71(4):1635-41. http://dx.doi.org/10.1590/0034-7167-2017-0648.

Lied, E. B., Magalhães, A. P., Soares, D. F., Trevisa, A. P. \& Marejon, C. F. M. (2019). Sustentabilidade Ecológica pelo cálculo da Pegada Ecológica. Revista Ambiência. 15(1). https://revistas.unicentro.br/index.php/ambiencia/article/view/4312.

Luis, H. P., Assunção, V. A. \& Luis, L. F. S. (2012). Tradução e validação para português de um questionário de hábitos, conhecimentos e atitudes de saúde oral dos adolescentes. Revista Adolescência \& Saúde, 9(1), 7-11, https://repositorio.ul.pt/handle/10451/34276.

Magalhães Júnior, C. A. O., Corazza, M. J., Kiouranis, N. M., Araújo, M. C. P., Oliveira, A. L., Maricato, F. E., Schneider, E. M., Ortiz, A. J., Carmo, T., Carvalho, J. L., Araújo, J. L. D., Rangel, M., Gimenes, E. R. \& Carvalho, G. S. (2020). Concepções e representações sociais de professores sobre a sua formação inicial: construção e validação de um questionário. Revista ensaio pesquisa em educação em ciências. 22, Belo Horizonte. http://dx.doi.org/10.1590/21172020210110.

Marques, R. \& Xavier, C. R. (2019). O desenvolvimento do senso analítico no processo de ensino e aprendizagem na Educação Ambiental. RELACult - Revista Latino-Americana de Estudos em Cultura e Sociedade, 5(2). http://dx.doi.org/10.23899/relacult.v5i2.1393. 
Marques, R. \& Xavier, C. (2020). Alfabetização científica no ensino de ciências: numa sequência didática sobre a pegada ecológica do lixo. Revista de Ensino de Ciências e Matemática, 11(2), 84-106. https://doi.org/10.26843/rencima.v11i2.2504.

Meyer, M. L. (2018). Educação Ambiental a partir da identidade ecológica: um estudo de caso. 101 f. Dissertação. Programa de Pós-Graduação em ensino de Ciências - Mestrado Profissional. Universidade Federal de Pelotas, http://guaiaca.ufpel.edu.br/handle/prefix/4455.

Moro, R. de C. L. \& Paulino, S. R. (2020). Sustentabilidade na cadeia de suprimentos do varejo de vestuário: proposta de ampliação de requisitos ambientais em um programa setorial de boas práticas. Revista Gestão \& Sustentabilidade Ambiental, 9. http://dx.doi.org/10.19177/rgsa.v9e02020847-867.

Mota, F. dos S., Silva, G. V., Duarte, E. R., Machado, V. M., \& Soares, F. I. L. (2020). Percepção dos acadêmicos do curso de Administração sobre a Educação Ambiental na Universidade Federal do Oeste do Pará, Campus Alenquer. Research, Society and Development, 9(10), e2259108549. https://doi.org/10.33448/rsdv9i10.8549.

Natalli, L H., Munaretto, L. F., Bianchini, D. C. \& Henkes, J. A. (2020). Práticas de sustentabilidade ambiental em propriedades rurais. Revista gestão sustentabilidade ambiental, 9(1), 351-374. http://dx.doi.org/10.19177/rgsa.v9e12020351-374.

Nobre, É. B. (2012). Elaboração e validação de questionário para descrever o estilo de vida de mães de pré-escolares. Dissertação. 91 fls. Programa de Pediatria. Faculdade de Medicina da Universidade de São Paulo.

Nunes, M. E. R.; França, L. F., \& Paiva, L. V. de. (2017). Eficácia de diferentes estratégias no ensino de educação ambiental: associação entre pesquisa e extensão universitária. Ambiente \& Sociedade, 20(2), 59-76. https://dx.doi.org/10.1590/1809-4422asoc228r1v2022017.

Nunes, A. G., Sousa, L. A., Cruz, F. N. I., Vale, M. R. L. \& Pontes Junior, J. A. F. (2020). Validação de conteúdo de um instrumento para mensuração do conhecimento sobre musculação, alimentação e suplementação. Research, Society and Development, 9(2).

Oliveira e Aguiar, A.; Ribeiro, C. S.; \&Nascimento, A.P. B. (2018). Percepção ambiental de Trabalhadores em Empresa Certificada ISO 14001: práticas e comportamentos. Desenvolvimento em questão. (45), 316-335. http://dx.doi.org/10.21527/2237-6453.2018.45.316-335.

Oliveira, I. P. \& Brasil, D. do S. B. (2020). Psicologia ambiental e problemas ambientais: uma revisão de literatura. DOXA: Revista Brasileira de Psicologia e Educação, 22(1), 108-122. https://doi.org/10.30715/doxa.v22i1.13735.

Pasquali, L. (2009). Psicometria. Revista da Escola de Enfermagem da USP. 43, 992-999. https://doi.org/10.1590/S0080-62342009000500002.

Pasquali, L. (2010). Instrumentação psicológica: Fundamentos e prática. Artmed.

Pasquali, L. (2013). Psicometria: Teoria dos testes na Psicologia e na Educação. (5a ed.), editora vozes Petrópolis.

Paulista, C. R., Cavadas, L. S., Santos, R. A., Santos, W. A. \& Erthal Junior, M. (2018). Avaliação ambiental de uma instituição de ensino a partir do uso de indicadores de sustentabilidade. Brazilian Journal of Development, 4(5), 1955-1979.

Pedreira, R. B. S., Rocha, S. V., Santos, C. A., Vasconcelos, L. R. C. \& Reis, M. C. (2016). Validade de conteúdo do Instrumento de Avaliação da Saúde do Idoso. Revista Einstein, 14(2), 158-77. 10.1590/S1679-45082016AO3455.

Pimentel, T. S. (2018). Construção e validação do instrumento para consulta de enfermagem ao indivíduo com diabetes mellitus tipo 2 . Dissertação. 130 fls. Mestre em Enfermagem, Universidade Federal de Sergipe.

Ribeiro, C. da S., Coutinho, C. \& Boer, N. (2020). Saber e complexidade como fundamento epistemológico da pedagogia e da cidadania ambiental. 21(1). Revista Eletrônica Disciplinarum Scientia: https://doi.org/10.37780/dsch.v21n1-016.

Roisemam, M. de M. L. (2008). Elaboração e validação de um questionário para avaliar conhecimento de pediatra e médicos de família na prevenção da doença cardiovascular na infância. Dissertação de Mestrado. Instituto Fernandes Figueira, Rio de Janeiro.

Royer, M. R. \& Petik, V. C. F. (2019). Pegada ecológica: que marcas os estudantes de biologia de uma universidade pública brasileira deixarão no planeta? Biografía, Escritos sobre la Biología y su Enseñanza. 672-682. https://revistas.pedagogica.edu.co/index.php/bio-grafia/article/view/10965.

Santos, F. C. dos. (2016). Construção e validação semântica de um instrumento para avaliação de competências de enfermeiros que atuam em oncologia. Dissertação. 150p. Escola de Enfermagem de Ribeirão Preto da Universidade de São Paulo.

Santos, L. R. O., Costa, J. de J., Melo \& Souza, R. (2020). Educação (Ambiental) para a cidadania: ações e representações de estudantes da Educação Básica. REMEA - Revista Eletrônica do Mestrado em Educação Ambiental, 37(1), 188-207. https://doi.org/10.14295/remea.v37i1.9678.

Schwantz, P. I., Becker, G. A., Etges, T., Roth, J. C. G., \& Lara, D. M. (2019). Análise da satisfação dos agricultores integrantes do programa "protetor das águas" no município de Vera Cruz/RS. Revista gestão e sustentabilidade ambiental, 8(4), 552-566.

Siqueira, M. \& Marques, D. F. (2018). Desenvolvimento e validação do instrumento de compreensão de expressões idiomáticas. Revista de estudos da linguagem, 26(2), 571-591. http://dx.doi.org/10.17851/2237-2083.26.2.571-591.

Silva, V. J. M O., Vizzotto, P. A., Martins, S. N. \& Forneck, K. L. (2020). Elaboração e validação do instrumento de medida do conhecimento empreendedor básico. Research, society and development, 9(7). http://dx.doi.org/10.33448/rsd-v9i7.4288.

Souza, A. C., Alexandre, N. M. C. \& Guirardello, E. de B. (2017). Propriedades psicométricas na avaliação de instrumentos: avaliação da confiabilidade e da validade. Epidemiologia e Serviços de Saúde, 26(3), 649-659. http://dx.doi.org/10.5123/s1679-49742017000300022 .

Torres, C. \& Fermam, R. K.S. (2016). Avaliação da competição no mercado de carbono: elaboração e validação de questionário. Revista Internacional de Ciências, 6(2), 199-213. https://doi.org/10.12957/ric.2016.23501. 
Research, Society and Development, v. 10, n. 6, e6310615542, 2021

(CC BY 4.0) | ISSN 2525-3409 | DOI: http://dx.doi.org/10.33448/rsd-v10i6.15542

Turmina, E., Kanieski, M. R., Jesus, L. A., Rosa, L. H., Batista, L. G., \& Almeida, A. N. (2018). Avaliação de impactos ambientais gerados na implantação e operação de subestação de energia elétrica: um estudo de caso em Palhoça, SC. Revista de Ciências Agroveterinárias, 17(4). https://doi.org/10.5965/223811711732018589.

Vilarinho, L. R. G. \& Monteiro, C. C. R. (2019). Projetos de educação ambiental escolar: uma proposta de avaliação. Revista brasileira de educação ambiental (REVBEA), 14(1), 439-455.

Vizzotto, P. A. \& Mackedanz, L. F. (2019) Física aplicada ao trânsito: processo de validação de um questionário para coleta de dados. Revista Brasileira de Ensino de Física. 41(3). http://dx.doi.org/10.1590/1806-9126-RBEF-2018-0256.

Wackernagel, M.; Rees, W. (1996). Our ecological footprint: reducing human impact on the earth. Gabriola Island, New Society Publishers, 167 p.

Zacarias, E. F.. \& Higuchi, M. I. G. (2017). Relação pessoa-ambiente: caminhos para uma vida sustentável. INTERAÇÕES, 18(3), 121-129. http://dx.doi.org/10.20435/inter.v18i3.1431. 